\title{
Impact of sensitization workshop on knowledge regarding tuberculosis among final year medical students
}

\section{Purushottam A. Giri, Deepak B. Phalke}

Department of Community Medicine (PSM), Rural Medical College and Pravara Rural Hospital of Pravara Institute of Medical Sciences (Deemed University),

Loni, Maharashtra, India

Address for the Correspondence:

Dr. Purushottam A. Giri, Associate Professor, Department of Community Medicine (PSM),

Rural Medical College, Loni, Maharashtra - 413736 , India. E-mail: drpgiri14@gmail.com

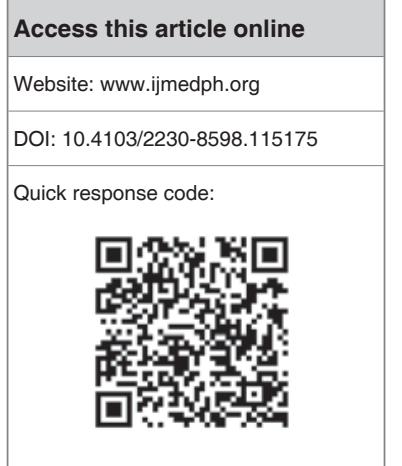

Background: India is the highest TB burden country in the world and accounts for nearly one fifth $(20 \%)$ of global burden of tuberculosis. Every year, approximately 1.8 million persons develop tuberculosis, of which about 8,00,000 are infectious and until recently, 3,70,000 cases died of it annually. In India, today, two deaths occur every three minutes from tuberculosis, but these deaths can be prevented with proper care and treatment. Materials and Methods: An interventional study was conducted from January to March 2009 by interviewing 86 final year medical students studying at Rural Medical College, Loni, India. A pre-designed and pre-tested questionnaire was used to collect the pre-workshop and post-workshop data. Questions were based on the knowledge regarding tuberculosis. Results were analyzed statistically using percentage, proportions, and Chi-square test. Results: In this study, about $55(63.9 \%)$ participants correctly specified RNTCP objectives in pre-test, while in post-test, $80(93.1 \%)$ participants could state correct response $(P<0.0001)$. In pre-test, $57(66.3 \%)$ participants thought that family member can be a DOTS provider, while during post-test, $80(93.1 \%)$ participants realized that DOTS should not be given by family member $(P<0.0001)$. Similarly, highly significant difference in pre- and post-test was observed for DOTS components. Conclusion: Sensitization workshops increase the knowledge of tuberculosis amongst the medical students. RNTCP training should be a part of regular activity of all medical colleges.

Key words: Knowledge, medical students, revised national tuberculosis control program, tuberculosis

\section{INTRODUCTION}

Tuberculosis (TB) is a major public health concern worldwide. The global burden of tuberculosis remains alarming despite the World Health Organization (WHO) and various national programs efforts. WHO recognizes the importance of tuberculosis-related knowledge, attitude and practice surveys in advocacy, communication, and social mobilization strategy planning. Despite good Directly Observed Treatment-Short Course (DOTS) coverage, India still accounts for about one-third of the world's TB burden and is one of the 22 high-burden countries identified by WHO. ${ }^{[1]}$ Even though the treatment success rate has tripled from $25 \%$ to $87 \%$ and death rate has declined from $29 \%$ to $5 \%$, it is still a major cause of morbidity and mortality in India. ${ }^{[2]}$ The National Tuberculosis Control Program (NTCP) was reviewed in India in 1992, and Revised National Tuberculosis Control Program (RNTCP) was drawn and formally launched in March 1997 with phased coverage in various states throughout India. ${ }^{[3]}$ Information, Education, and Communication (IEC) are an integral and important strategy of the program to create awareness among public, health care providers, and policy makers. The medical students are the doctors in making who hold the promise of providing health care services to the community in near future. Hence, the present study was conducted to assess the impact of sensitization workshop on knowledge regarding tuberculosis among final year medical students.

\section{MATERIALS AND METHODS}

This pre- and post-test interventional study was conducted from January 2009 to March 2009. A total 112 final year medical students (III MBBS, IV th term) studying at Rural Medical College, 
Loni, Maharashtra, India who were willing to participate in the study were included. However, $92(82.1 \%)$ students consented to participate in the study, and $6(6.5 \%)$ questionnaire from students could not be included for statistical analysis because of the lack of sufficient information. Hence, the total 86 medical students from final year (III MBBS, IV $^{\text {th }}$ term) were included for the study. A pre-designed and pre-structured questionnaire was used to collect the pre-workshop and post-workshop data. The questionnaire was pre-tested on subsample of 25 students and modified and necessary changes were made accordingly. The language of the questionnaire was English and contained 12 questions, which were based on the knowledge regarding tuberculosis. The questions covered knowledge of students about diagnosis, management, and strategies of revised national tuberculosis control program. The ethics committee of the institute approved the study. The sensitization workshop for one day was conducted in the auditorium of Medical College, consisted of series of lectures and audio-visual presentations followed by question-answer session covering all aspects of tuberculosis mentioned in questionnaire. Post-workshop data was collected. Results were analyzed statistically using percentage, proportions and Chi-square test was applied. Statistical significance was set at $P \leq 0.05$.

\section{RESULTS}

It was seen from Table 1 that there was significant improvement in knowledge about tuberculosis among students after sensitization workshop $(P<0.0001)$. Thirty-four $(39.5 \%)$ participants defined multi-drug-resistant tuberculosis (MDR-TB) precisely in pretest, while in post-test, $82(95.3 \%)$ participants could define it rightly. After workshop, the students knowledge about contraindication of anti-TB drug increased from $69.7 \%$ to $94.2 \%$, and $80(93.1 \%)$ students realized that DOTS should not be given by family member $(P<0.0001)$. The intensification of ACSM activities is an essential component of RNTCP. Only 52.3\% students knew about the full form of ACSM correctly, but after workshop, 93.1\% were able to give the full form of ACSM accurately $(P<0.0001)$. Post-workshop, $94.2 \%$ students became aware about the reagents are used in Ziehl-Neelsen staining in tuberculosis, and 78 (90.7\%) knew that INH prophylaxis should be given to breastfeeding babies whose mother have active tuberculosis $(P<0.0001)$.

\section{DISCUSSION}

In the present study, despite RNTCP being part of their undergraduate curriculum, there was inadequate knowledge about tuberculosis amongst undergraduates. This may be due to the facts that textbooks are not updated frequently and may not reflect current diagnostic and treatment guidelines. Another source of confusion is due to different regimens given in textbooks of pharmacology, community medicine, and general medicine. As medical education and public health are separate departments, change in policy of national program may not reach medical colleges. Our findings are also similar to those of several other investigators who have conducted surveys among health profession students. Though the assessment tool used may be different in different studies, the key core knowledge components might be same in all. A study done by Kilicaslan et al., ${ }^{[4]}$ evaluated undergraduate training on tuberculosis at Istanbul Medical School among fourth-year medical students $(n=828)$ and found many incorrect answers. Similarly, Bai et al. ${ }^{[5]}$ administered a survey to final year medical students in Hunan province of China, Rajpal et al.,,$^{[6]}$ among interns in Delhi, Hong et al., ${ }^{[7]}$ among general practitioners, and Teixeira et al., ${ }^{[8]}$ among medical students in Brazil came to similar conclusions. In contrast, Emili et al., ${ }^{\left[{ }^{[]}\right.}$assessed final year medical students from Canada, India, and Uganda found that the TB knowledge base and practice competency was adequate.

The present study showed statistically significant improvement in participant's knowledge of tuberculosis from pre-test to post-test. Similarly, study carried out in public health workers and DOTS workers by PS et al., ${ }^{[10]}$ and among high school children by

\begin{tabular}{|c|c|c|c|c|c|c|}
\hline \multirow[t]{2}{*}{ Particulars } & \multicolumn{2}{|c|}{ Pre-workshop (\%) } & \multicolumn{2}{|c|}{ Post-workshop (\%) } & \multirow[t]{2}{*}{$\chi^{2}$ value } & \multirow[t]{2}{*}{$P$ value } \\
\hline & Yes & No & Yes & No & & \\
\hline What is primary objective of RNTCP? & $55(63.9)$ & $31(36.1)$ & $80(93.1)$ & $06(6.9)$ & 21.52 & 0.000 \\
\hline What are five components of DOTS? & $50(58.1)$ & $36(41.9)$ & $82(95.3)$ & $04(4.7)$ & 33.35 & 0.000 \\
\hline $\begin{array}{l}\text { How many sputum samples required for diagnosis of } \\
\text { smear-positive case in RNTCP? }\end{array}$ & $75(87.2)$ & $11(12.8)$ & $83(96.5)$ & $03(3.5)$ & 4.97 & 0.026 \\
\hline $\begin{array}{l}\text { Sputum specimen should be collected and examined by } \\
\text { microscopy not later than- }\end{array}$ & $39(45.4)$ & $47(54.6)$ & $75(87.2)$ & $11(12.8)$ & 33.71 & 0.000 \\
\hline Family member can be a DOT provider & $57(66.3)$ & $29(33.7)$ & $80(93.1)$ & $06(6.9)$ & 18.97 & 0.000 \\
\hline Treatment regimen for smear-positive case in RNTCP- & $52(60.5)$ & $34(39.5)$ & $79(91.9)$ & $07(8.1)$ & 23.34 & 0.000 \\
\hline Which anti-TB drug is contraindicated in pregnancy? & $60(69.7)$ & $26(30.2)$ & $81(94.2)$ & $05(5.8)$ & 17.35 & 0.000 \\
\hline In RNTCP, ACSM stands for- & $45(52.3)$ & $41(47.7)$ & $80(93.1)$ & $06(6.9)$ & 35.86 & 0.000 \\
\hline Which reagents are used in Ziehl-Neelsen staining? & $30(34.9)$ & $56(65.1)$ & $81(94.2)$ & $05(5.8)$ & 66.07 & 0.000 \\
\hline What is Multi-Drug resistant tuberculosis (MDR-TB)? & $34(39.5)$ & $52(60.5)$ & $82(95.3)$ & $04(4.7)$ & 61.00 & 0.000 \\
\hline AFB can be seen under microscope with & $56(65.1)$ & $30(34.9)$ & $80(93.1)$ & $06(6.9)$ & 20.23 & 0.000 \\
\hline $\begin{array}{l}\text { INH prophylaxis should be given to breast feeding } \\
\text { babies whose mother have active tuberculosis }\end{array}$ & $28(32.5)$ & $58(67.5)$ & $78(90.7)$ & $08(9.3)$ & 61.46 & 0.000 \\
\hline
\end{tabular}

RNTCP=Revised national tuberculosis control program, DOTS=Directly observed treatment-short, MDR-TB=Multi-drug-resistant tuberculosis, AFB= Acid -fast bacilli; INH=Isoniazid, $(n=86)$ 
Gopichandran et al.. ${ }^{[11]}$ found statistically significant improvement in knowledge regarding tuberculosis from pre-test to post-test.

A study done by Nakanishi et al., ${ }^{[12]}$ in a teaching hospital in Japan, between 1999 and 2001, a total of 2159 physicians, nursing staff, medical technicians, pharmacists, and medical and dental students completed a questionnaire on their interest in and knowledge of TB. Fewer than $50 \%$ of participants, including physicians, correctly answered basic questions about TB. It concluded that employees and students in this university hospital do not have enough knowledge about TB, and that the strengthening of health education on TB would be necessary. Our study corroborates these findings in the sense that an effort is needed to improve student's knowledge about tuberculosis.

\section{Limitations}

Student's attitude and practices were not evaluated. As group was defined as final year medical students, there was no scope to increase the sample size.

\section{CONCLUSION}

The present study revealed inadequacies in the knowledge of tuberculosis amongst undergraduate students. As tuberculosis continues to be a major public health problem in India, our medical graduates must be competent in diagnosis and treatment and RNTCP guidelines. There is change in diagnostic and treatment guidelines from time to time, e.g., duration of cough for TB suspect, categorization under DOTS etc., which may not be reflected in textbooks immediately. Periodic RNTCP sensitization should be the part of regular activity in all medical colleges.

\section{ACKNOWLEDGMENT}

We express our deep sense of gratitude to the Management, Pravara Medical Trust, and The Principal, Rural Medical College Loni, Maharashtra, India. We also acknowledge the help of Dr. Pallavi R. Shidhaye, Tutor, Dept. of Community Medicine (PSM) and Medical interns- Parul Arora and Purva Mehta.

\section{REFERENCES}

1. World Health Organization. Global Tuberculosis Control-Surveillance, Planning, Financing, 2011. Available from: http://www.who.int/tb/ publications/global_report/en/. [Last accessed on 2012 Jan 17].

2. TB India 2010. RNTCP Status Report. Central TB Division, Ministry of Health and Family Welfare. New Delhi: Nirman Bhavan; 2010.

3. Sunderlal. Textbook of Community Medicine. $2^{\text {nd }}$ ed. India: Satish Kumar Jain; 2009. p. 424.

4. Kilicaslan Z, Kiyan E, Erkan F, Gurgan M, Aydemir N, Arvesen O. Evaluation of undergraduate training on tuberculosis at Istanbul Medical School. Int J Tuberc Lung Dis 2003;7:159-64.

5. Bai LQ, Ziao SY, Xie HW, Yang GF, Wang YZ. Knowledge and practice regarding tuberculosis among final-year medical students in Hunan, China. Zhonghua Jie He He Hu Xi Za Zhi 2003;26:458-46.

6. Rajpal S, Mittal A, Dhingra VK, Malhotra R, Gupta R, Malhotra C, et al. Knowledge, attitude and practices regarding tuberculosis and dots among interns in Delhi. India J Coll Physicians Surg Pak 2007;17:457-61.

7. Hong YP, Kwon DW, Kim SJ, Chang SC, Kang MK, Lee EP, et al. Survey of knowledge, attitude and practices for tuberculosis among general practitioners. Tuber Lung Dis 1995;76:431-5.

8. Teixeira EG, Menzies D, Cunha AJ, Luiz RR, Ruffino-Netto A, Scartozzoni MS, et al. Knowledge and practices of medical students to prevent tuberculosis transmission in Rio de Janeiro, Brazil. Pan Am J Public Health 2008;24:265-70.

9. Emili J, Norman GR, Upshur RE, Scott F, John KR, Schmuck ML. Knowledge and practices regarding tuberculosis: A survey of final-year medical students from Canada, India and Uganda. Med Educ 2001;35:530-6.

10. Wu PS, Chou P, Chang NT, Sun WJ, Kuo HS. Assessment of Changes in Knowledge and Stigmatization Following Tuberculosis Training Workshop in Taiwan. J Formos Med Assoc 2009;108:377-85.

11. Gopichandran V, Roy P, Sitaram A, Karthick, John K. Impact of a simple educational intervention on the knowledge and awareness of tuberculosis among high school children in Vellore, India. Indian J Community Med 2010;35:174-5.

12. Nakanishi $\mathrm{Y}$, Izumi M, Abe $\mathrm{K}$, Harada $\mathrm{T}$, Inoue $\mathrm{K}$, Wataya $\mathrm{H}$, et al. Questionnaire about impression and knowledge of tuberculosis in employees and students in a university hospital. Kekkaku 2002;77:457-63.

How to cite this article: Giri PA, Phalke DB. Impact of sensitization workshop on knowledge regarding tuberculosis among final year medical students. Int J Med Public Health 2013;3:100-2.

Source of Support: Nil, Conflict of Interest: None declared. 